2005-09-01

\title{
Interaction of Carbon Nanotubes with Sugar Complexes
}

\author{
Alan Casey \\ Technological University Dublin, alan.casey@tudublin.ie \\ Gordon Farrell \\ Technological University Dublin, gordon.farrell@tudublin.ie \\ Mary McNamara \\ Technological University Dublin, Mary.McNamara@tudublin.ie
}

See next page for additional authors

Follow this and additional works at: https://arrow.tudublin.ie/nanolart

Part of the Physics Commons

\section{Recommended Citation}

Casey, A. et al. (2005) Interaction of carbon nanotubes with sugar complexes. Synthetic Metals, vol. 153, Issues 1-3, pp 357-360. 21 September. doi:10.1016/j.synthmet.2005.07.298

This Article is brought to you for free and open access by the NanoLab at ARROW@TU Dublin. It has been accepted for inclusion in Articles by an authorized administrator of ARROW@TU Dublin. For more information, please contact arrow.admin@tudublin.ie, aisling.coyne@tudublin.ie,gerard.connolly@tudublin.ie. Funder: Technological Sector Strand 1 funding. 


\section{Authors}

Alan Casey, Gordon Farrell, Mary McNamara, Hugh Byrne, and Gordon Chambers

This article is available at ARROW@TU Dublin: https://arrow.tudublin.ie/nanolart/2 


\title{
Interaction of Carbon Nanotubes with Sugar Complexes
}

\author{
A. Casey ${ }^{1,2}$, G.F. Farrell ${ }^{2}$, M. McNamara ${ }^{2,3}$, H.J. Byrne ${ }^{1,2}$ and G. Chambers ${ }^{1,2}$. \\ ${ }^{I}$ School of Physics, Dublin Institute of Technology, Kevin Street, Dublin 08, Ireland \\ ${ }^{2}$ FOCAS, Dublin Institute of Technology, Kevin Street, Dublin 08, Ireland \\ ${ }^{3}$ School of Chemistry, Dublin Institute of Technology, Kevin Street, Dublin 08, Ireland
}

\begin{abstract}
The interaction of carbon nanotubes with soft organic molecules such as cyclodextrins and other saccarides has recently been shown to produce water soluble composites. Such systems offer considerable advantages over polymer based composites due to their biocompatibility and noncovalent coupling which can potentially preserve the unique properties of the tubes. The mechanism of interaction for such systems has been proposed to be dominated by hydrophobic and hydrophilic interactions along the surface of the tube. However efforts to characterise and rationalise such noncovalent interactions between the sugar-based materials and the carbon nanotubes have been slow to emerge. In this study a composite system has been formed using HiPco Single Walled Carbon Nanotubes (HCNT) and starch (extracted from rice). This composite was characterised using a range of spectroscopic techniques, which showed clear evidence of an intermolecular interaction between the HCNT and starch. The characterisation of these systems will be presented and evidence to support the notion of a noncovalent interaction is clear.
\end{abstract}

Keywords: Fullerenes and derivatives, UV-Vis-NIR absorption, Infrared and Raman Spectroscopy

\section{Introduction}

The interaction of carbon nanotubes with soft organic molecules has begun to emerge as an accepted route towards the purification and isolation of this unique fullerene based material. While experiments performed using polymeric and surfactant based routes have shown considerable efficiencies for the purification and dispersion of single walled carbon nanotubes (SWNT) [1]. Much of the work has focused on using harsh solvent based systems with poor affinities for biological systems. The report by Bandyopadhyaya et al [2] that gum Arabic, a polysaccharide, could form stable well dispersed suspensions of SWNT in aqueous solution excited the entire area with the potential that such composite systems could hold the key to the successful integration of nanotubes into biological environments. The interaction of saccharides with fullerenes was demonstrated as early as 1993 when a $\mathrm{C}_{60}-\gamma$ cyclodextrin complex was formed [3]. It has since been suggested that these oligosaccharides interact with the SWNT via a Van der Waals interaction. Furthermore it is proposed that these soft organic materials can actually cut the nanotube although no specific mechanism was proposed. It was however shown

*Corresponding author. Tel: +353-1-4027932; fax: +353-1-4029999 E-mail: alan.casey@dit.ie spectroscopically that the interaction was along the length of the tube and appeared to introduce a compressive strain on the tube surface consistent with a plausible cutting effect [4]. Most recently amylose a polymeric form of glucose and a constituent of starch has been proposed to weakly interact with HiPco Carbon Nanotubes (HCNT) via non-specific hydrophobic interactions [5]. Although spectroscopic evidence for such interactions was weak, thermal analysis and microscopy did indicated the formation of a starch-nanotube complex. In this study further spectroscopic analysis is carried out on a similar starch system to confirm its potential as a dispersing agent for carbon nanotubes. Work carried out by Star et al [6] proposed an enhanced interaction of amylose and HCNT by creating an inclusion complex of amylose and excess iodine prior to the addition of the HCNT creating a coiled starch-nanotube complex. The complex formed in this study replaced the iodine with bromine resulting in the formation of a tertiary composite of amylose, bromine and HCNT.

\section{Experimental}

In this Study HCNT were used to form two complexes with starch. The first simple complex of starch and nanotubes was created by dispersing HCNT $(1 \mathrm{mg})$ in $25 \mathrm{ml}$ of a starch solution $(10 \mathrm{~g} / \mathrm{l})$ followed by ultrasonication (4 minutes medium power). This system was essentially used as a 
control to investigate the effects of the bromine on the spectroscopic characteristics of the starch HCNT complex. A solution of the starch $(10 \mathrm{~g} / \mathrm{l})$ was saturated with bromine, which resulted in an inclusion complex of the starch being formed prior to the addition of the HCNT. The brominestarch solution was allowed to settle before the supernatant was decanted off thereby limiting any potential effects of excess or unreacted bromine. This solution was then used to form the nanotube complex. Upon the addition of the nanotube the solution under went a process involving sonication and centrifugation [6]. Both these complexes were then characterised using a variety of spectroscopic techniques. Vibrational analysis was carried out on dropcast films using an Instruments S.A LabRam 1B Raman microscope and a Perkin Elmer Spectrum GX. Electronic analysis was carried out again on dropcast films the use of a Perkin Elmer Lamada 900 UV/VIS/NIR spectrometer. Circular dichroism measurements were carried out directly on the suspensions using a Jasco J-810 spectropolarimeter.

\section{Results and Discussion}

Initially it was visually noted that both methods resulted in the formation of a water-soluble complex of differing colour. The first complex of amylose and HCNT resulted in the formation of a clear solution, which held the HCNT in suspension for approximately three weeks. After this time period difficulties arose in the detectably of the HCNT in the samples, suggesting that the amylose suspensions are not stable and a gradual settling out process is occurring. It is postulated that amylose, a linear polymer of 1-4 linked $\alpha-$ $\mathrm{D}$ glucose units which resides in a left handed helical arrangement in an aqueous environment, holds the HCNT in suspension by hydrophobic-hydrophilic interactions with the nanotube as has been previously reported [5] however the interaction is not optimised due to the nature of the amlyose coil in aqueous solution. The second complex formed uses bromine to form a more ordered helical arrangement of amylose by inclusion prior to the addition of the HCNT. The resultant complex permitted the HCNT to remain in solution for up to the lifetime of the starch in solution (i.e. six weeks refrigerated) and retained the characteristic orange colour of the bromine amylose inclusion complex. This is in stark contrast to the complex formed with amylose alone and the complex formed by Star et al [6] (which used iodine as opposed to bromine). Both of these resulted in clear colourless solutions. It was proposed by Star et al [6] that the iodine is completely displaced by the inclusion of the nanotubes into the cavity of the starch thereby rendering the solution colourless. As a result the colour retention of the complex here may be an indication that all the bromine is not displaced but rather due to its smaller size can be readily accommodated in the cavity with the nanotubes forming a tertiary complex or composite. Of course the possibility that the colour may the result of excess bromine cannot be ruled out. However the volume of bromine used to produce the initial starch inclusion complex in successive runs was significantly reduced, yet the orange colour was still retained upon the addition of the tubes.

Due to the nature of the starch, bromine, HCNT complex (orange colour) electronic analysis was used to verify the presence of HCNT. The absorption spectrum of HCNT (Fig.1a) consists of three broad absorption peaks centred approximately at $1200 \mathrm{~nm}, 800 \mathrm{~nm}$ and $270 \mathrm{~nm}$. The peaks at $1200 \mathrm{nmn}$ and $800 \mathrm{~nm}$ being attributed to optical transitions between mirror image spikes in the density of states, with the feature at $270 \mathrm{~nm}$ attributed to the $\pi$-plasma frequency of $5 \mathrm{eV}$ in carbon materials [7], these features were present in the complex spectrum verifying the presence of HCNT. Using the broad peak in the NIR region (Fig.2.d) the diameter distribution [8] of the HCNT used was estimated to be $0.8 \mathrm{~nm}-1.36 \mathrm{~nm}$.

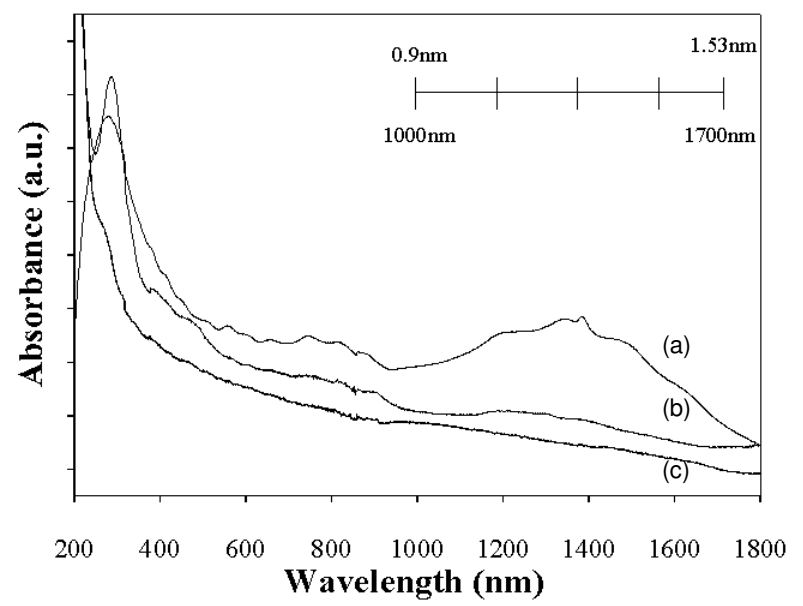

Fig.1. UV/VIS/NIR Absorption Spectra of dropcast (a) Raw HCNT (b) Starch, Bromine, HCNT Complex (c) Starch.

In comparison, the absorption spectrum of the starch (Fig.2.c) appears to be relatively featureless and a similar spectrum to this was obtained for the starch nanotube complex. Figure 2 (b) shows the absorption spectrum of the starch-bromine- HCNT complex. It can be seen there are HCNT features present in the NIR region which indicates some degree of selectivity towards the mid to lower range of the tube bundles present in the pristine sample further suggesting a specific interaction. However the spectra do not contain any definitive evidence of the formation of a true chemical complex but rather indicates the presence of HCNT in the sample.

Further electronic analysis was sought using circular dicroism. Preliminary results indicate a shift of $\sim 22 \mathrm{~nm}$ in the predominant feature of starch centred at $\sim 300 \mathrm{~nm}$ which may be evidence of an inclusion complex. Indeed such shifts in circular dicroisim peaks are regularly used as an indication of the degree of inclusion in guesthost systems [9]. However the exact nature of such complexes is often difficult to ascertain for electronic spectroscopic techniques. In general the results obtained 
from electronic spectroscopy showed little evidence to support the formation of a complex. As a result vibrational analysis was carried out to further elucidate the nature of the complex.

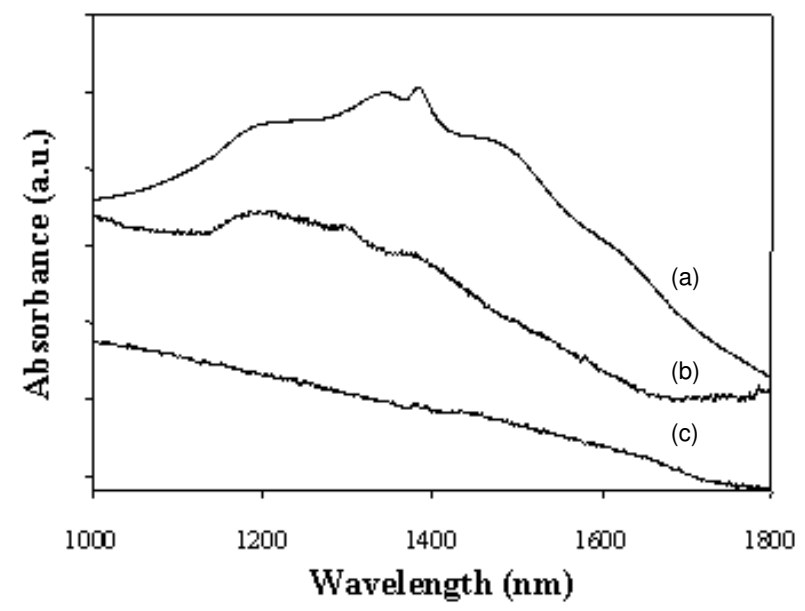

Fig.2. NIR Absorption Spectra of dropcast (a) Raw HCNT (b) Starch, Bromine, HCNT Complex (c) Starch (Note: b and c multiplied by a factor of 10)

Infrared absorption spectroscopy was carried out on all samples to probe their vibrational character. The FTIR spectrum of carbon nanotubes (not shown) agreed with literature [10] consisting of three main features at $1600 \mathrm{~cm}^{-1}, 1400 \mathrm{~cm}^{-1}$ and $1180 \mathrm{~cm}^{-1}$ which have been attributed to the $\mathrm{C}-\mathrm{C}$ and $\mathrm{C}=\mathrm{C}$ stretching along the hexagonal array of the nanotube backbone [10].

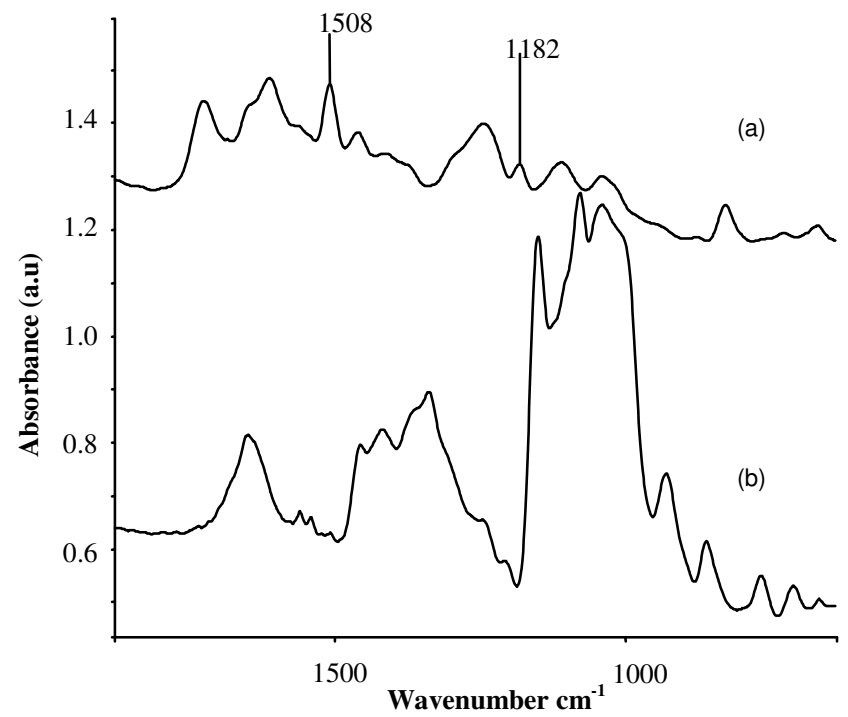

Fig.3. Infrared spectra (a) Starch-Bromine-HCNT Complex (b) Raw Starch

The infrared signal of starch (Fig.3b.) in contrast is extremely rich and contains a number of strong features primarily due to contributions from O-H, C-H C-C and C$\mathrm{O}$ stretching. FTIR analysis of the both complexes, showed mainly strong features of starch resulting from glucose with the exception of two new features at $1182 \mathrm{~cm}^{-1}$ and $1509 \mathrm{~cm}^{-1}$ in the bromine, starch, HCNT complex. The feature at $1182 \mathrm{~cm}^{-1}$ could be attributed to the C-C stretching in the nanotube. This again indicates that HCNT were present but gave no clear insight into the formation of a complex. The exact origin of the second feature at $1509 \mathrm{~cm}^{-1}$ is still unclear. The appearance of IR new features in complexes has in the past been attributed to a number of processes such as the relaxation of the selection rules, which allow otherwise forbidden modes of vibration to become IR active. However there is no evidence here that this mode is either a Raman active mode or a silent mode. Rather it is postulated that this feature is in fact a vibrational mode characteristic of the interaction between the nanotube and starch. As a result this mode is potentially the first spectroscopic evidence of true complex formation between the nanotubes and the starch. Further vibrational analysis was carried out using Raman spectroscopy, a technique known to be extremely sensitive to changes in the local environment of the carbon nanotubes.

Raman analysis was carried out on all samples at $514 \mathrm{~nm}$ laser excitation. The Raman spectrum of pristine nanotubes [11] (Fig.4.a) consists of three main features, the radial breathing mode, the $D$ line and the $G$ line. These modes are very sensitive to any perturbation to the local environment of the nanotube and are therefore give good indication of complex formation. In the complex formed with amylose only, there was no significant difference in the spectrum, all modes being present and their shapes and positions characteristic of that of the raw HCNTs, as had been previously reported [5]. This was not the case with the complex involving bromine as a precursor. As mentioned earlier all bromine was not removed in the formation of the complex as indicated by its final colour. For this reason it was postulated that a tertiary composite could have been formed of starch, bromine and HCNT.

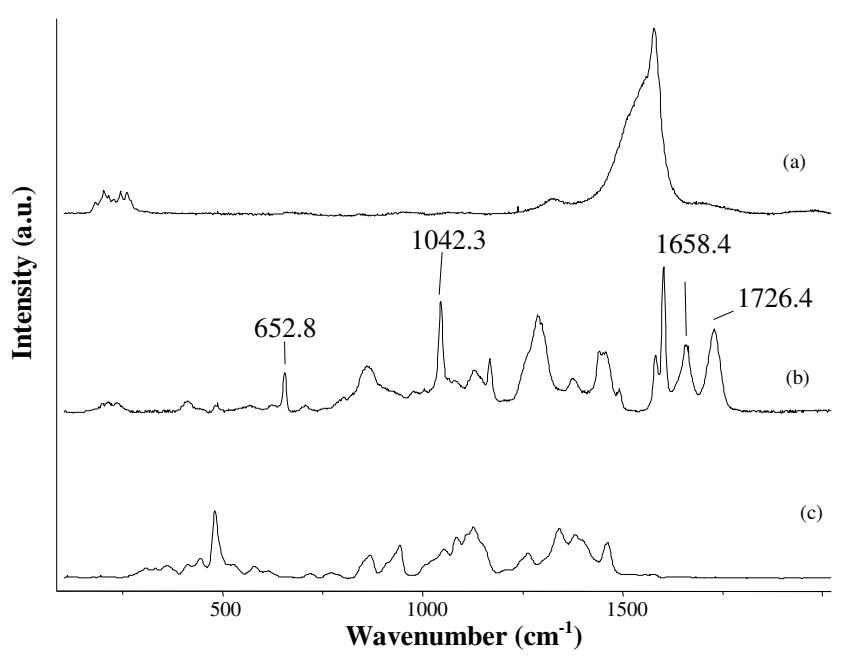

Fig.4. Raman Response for (a) Raw HCNT (b) Starch, Bromine, HCNT Complex (c) Raw Starch

Supportive evidence for this tertiary system can be seen in the Raman spectrum of the composite (Fig.4b.) by 
the appearance of four new features in the spectrum at $652.8 \mathrm{~cm}^{-1}, 1042.2 \mathrm{~cm}^{-1}, 1658.4 \mathrm{~cm}^{-1}$ and $1726.4 \mathrm{~cm}^{-1}$. It is important to note that this spectrum is not a direct summation of the starch and HCNT spectra. The new feature present at $1042 \mathrm{~cm}^{-1}$ may be due to IR active modes of the starch becoming Raman active due to changes in symmetry relaxing the normal selection rules.

Indeed close examination of the IR spectrum reveals a number of candidate FTIR modes, which could become Raman active in this region. The other three features however appear to be truly new features their presence undoubtedly indicating the formation of a new starch-bromine-Nanotube complex. Another noticeable feature of this spectrum is the narrowing of the $G$ line $_{a}$ with a shift in peak position from $1588 \mathrm{~cm}^{-1}$ in the raw sample to $1601 \mathrm{~cm}^{-1}$ in the complex. Similar up shifts in this mode have been reported before in cyclodextrin- nanotube complexes and were attributed to the absorption of the cyclodextrin along the length of the tube constricting the molecular vibrations of the SWNT. However it should be noted that the reduction in symmetry due to complex formation may also play an important role in determining the relative mode positioning.

Finally there are also noticeable differences in the radial breathing mode region of the spectrum of the bromine based complex. The RBMs of pristine nanotubes are inversely related to the diameters of the nanotubes [12] and using this relationship, a diameter range for the raw sample was calculated [12] to be from $0.8 \mathrm{~nm}-1.4 \mathrm{~nm}$. This was not the case in the complex formed involving bromine (Fig.5.b). In this spectral region starch is clear of features [13] (Fig.4.c), facilitating the diameter range of the complex to be calculated in a similar fashion to the raw sample. The calculated range of the complex had decreased to $1.00 \mathrm{~nm}-1.3 \mathrm{~nm}$, which is in broad agreement with the degree of selectivity noted from the absorption spectra.

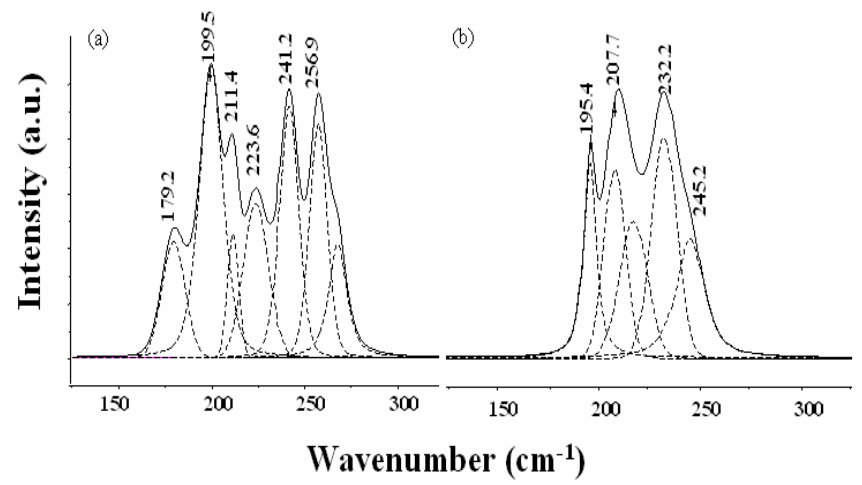

Fig.5. Raman response for the radial breathing mode (RBM) (a) Raw HCNT (b) Starch, Bromine, HCNT Complex.

In summary, the efficiency of starch to act a dispersion agent of HCNT in an aqueous solution was increased by the addition of bromine into the system as it facilitated the HCNT to remain in suspension for longer. Although electronic analysis showed little evidence to support the notion of a tertiary complex of starch, bromine and HCNT vibrational analysis provided confirmation of the formation of a true chemical complex by the appearance of the new features in the spectrum.

\section{Conclusions}

It has been shown spectroscopically that a tertiary composite system was created utilising a guest host mechanism of starch. Vibrational spectroscopy showed clear evidence of the formation of complex between the starch and the carbon nanotubes while evidence from the electronic spectroscopy was considerable weaker in it still provided clear signs of an intermolecular interaction. The degree of selectivity of these complexes is still the subject of much speculation and work is ongoing to determine the nature and extent of the interaction. Undoubtedly such composite systems do provide a potential route towards the bio functionalisation and integration of nanotubes into biological environments. However questions are raised about the insufficient displacement of bromine in the final complex

\section{Acknowledgements}

FOCAS is funded under the Irish Government National Development Plan 2000 - 2006 with assistance from the European Regional Development Fund. A. Casey acknowledges the Technological Sector Strand 1 funding.

\section{References}

[1] A.B. Dalton, C. Stephan, J.N. Coleman, B. McCarthy, P.M. Ajayan, S Lefrant, P. Bernier, W.J. Blau, H.J. Byrne, Journal of Physical Chemistry B, 104, 10012-10016 (2000)

[2] R. Bandyopadhyaya, E. Nativ-Roth, O. Regev and R. YerushalmiRozen, Nanoletters. 2 (2002) 25.

[3] T. Andersson et al., in "Electronic Properties of Fullerenes", H. Kuzmany, J. Fink, M. Mehring and S. Roth eds, Springer Series in Solid State Sciences, vol 117, Springer Verlag, Berlin Heidelberg (1993).

[4] G. Chambers, C. Carroll, G.F. Farrell, A.B. Dalton, M. McNamara, M. in het Panhuis, H.J. Byrne, Nanoletters, 3 (6): 843-846 (2003)

[5] Cheng-yi Lii, L. Stobinski, P. Tomasik, C. Liao, Carbohydrate Polymers. 51 (2002) 93-98.

[6] A. Star, D.W Steuerman, J.R. Heath and J.F. Stoddart, Angew. Chem. Int. Ed. (2002), 14, No.14.

[7] Physical Properties of Carbon Nanotubes, R. Saito, G. Dresselhaus, M.S Dresselhaus, Imperial college Press, (1998)

[8] Zhonghua Yu and Louis Brus, J. Phys. Chem. B 2001, 105, 1123-1134 [9] A. Rodger and B Nordén, Circular Dichroism and Linear Dichrosim, Oxford University Press, 1997.

[10] C. Branca, C. Corsaro, F. Frusteri, V. Magazu, A. Mangione, F. Migliardo and U. Wanderlingh, Diamond and Related Materials, 13 (2004) 1249-1253.

[11]M.S Dresselhaus, A. Jorio, A.G Souza Filho, G. Dresselhaus and R. Saito, Physica B 323 (2002) 15-20.

[12] A. Kukovecz, Ch. Kramberger, V. Georgakilas, M. Prato and H. Kuzmany, Eur. Phys. J. B 28 (2002), 223-230

[13]N. Dunpuy and J. Laureyns, Carbohydrate Polymers,49 (2002) 83-90. 
